\title{
Inhomogeneous LOFF phase revisited for surface superconductivity
}

\author{
Victor Barzykin and Lev P. Gor'kov \\ National High Magnetic Field Laboratory, Florida State University, \\ 1800 E. Paul Dirac Dr., Tallahassee, Florida 32310
}

\begin{abstract}
We consider 2D surface superconductivity in high magnetic fields parallel to the surface. We demonstrate that the spin-orbit interaction at the surface changes the properties of the inhomogeneous superconducting Larkin-Ovchinnikov-Fulde-Ferrell state that develops above fields given by the paramagnetic criterion. Strong spin-orbit interaction significantly broadens the range of existence of the LOFF phase, which takes the form of periodic superconducting stripes running along the field direction on the surface, leading to the anisotropy of its properties. In connection with experiments by J.H. Schön et al. [Nature 914, 434 (2001)] on superconductivity of electrically doped films of the cuprate material $\mathrm{CaCuO}_{2}$, we also discuss this problem for the d-wave pairing to indicate the possibility of a re-orientation transition as the magnetic field direction is rotated in the plane parallel to the surface. Our results provide a tool for studying surface superconductivity as a function of doping.
\end{abstract}

PACS numbers: 74.20.-z, 71.18.+y, 73.20.At, 76.60.Cq

There has been renewed experimental and theoretical interest in the properties of metallic states localized at a surface, and surface superconductivity (SSC). Surface states (a.k.a. Tamm's levels) are well-known from the physics of semiconductors. Numerous ARPES [1, 2] data and STM studies of Friedel oscillations 33 have now proven the existence of $2 \mathrm{D}$ metallic bands even at surfaces of metals. The bands are well-separated from the bulk and possess clear-cut 2D Fermi surfaces. For heavy enough elements such Fermi surfaces are split further by strong spin-orbit (SO) interactions. For example, $\mathrm{SO}$ energy for electrons at the Fermi level for $\mathrm{Au}$ is estimated at $0.1 \mathrm{eV}$ [1], while for Li-doped surfaces of Mo and $\mathrm{W}$ its value increases up to $0.13 \mathrm{eV}$ and $0.5 \mathrm{eV}$, correspondingly [2].

Islands of a surface superconducting phase were also observed for the surface-doped tungsten bronzes, $\mathrm{WO}_{3}: \mathrm{Na}$, at $T_{c}=91.5 \mathrm{~K}[\mathrm{~A}]$. The ARPES results mentioned above suggest that SSC may actually be a rather widespread phenomenon.

It is noteworthy that the bulk $\mathrm{WO}_{3}$ is an insulator at low doping $[$. Recently a remarkable breakthrough has been accomplished by doping insulator surfaces electrically in the so-called Field-Effect Transistor (FET) geometry [6]. SSC with high $\mathrm{T}_{c}$ was induced by both electronic and hole doping of films of the prototype cuprate material, $\mathrm{CaCuO}_{2}$ ].

All this makes us believe that the search for SSC emerges as a new and important development in studies of the properties of surfaces, especially their metallic properties. As for the SSC itself, its mechanisms are unknown, and may have nothing to do with the ones in the bulk. Of a special challenge is the possibility of superconductivity at surfaces of ordinary metals such as $\mathrm{Cu}$

\footnotetext{
*Also at L.D. Landau Institute for Theoretical Physics, Chernogolovka, 142432, Russia
}

[3] or lithium-doped Mo and W[2], and the influence of substrate on superconductivity in thin films. Thus, it is necessary to return to a more careful investigation of the low temperature properties of surfaces with absorbed atoms and the role of adsorbed atoms as dopants of carriers into the metallic surface zone (e.g., see Ref.[2]) like it was discovered for $\mathrm{WO}_{3}: \mathrm{Na}$ 画.

The major challenge to such a program lies in discerning SSC and studying its properties. Even for insulators where doping by FET, ideally, provides an effective control on surface properties, experimental tools to probe SSC are limited in numbers. The Meissner effect due to surface superconducting islands would probably never produce a bulk screening. Thermodynamical probes are also difficult because of the smallness of contributions from surface layers of atomic thicknesses. So far, e.g., in Ref. [6], surface superconductivity has only been detected by measuring resistivity dependence on temperature in the perpendicular-to-plane magnetic fields.

In what follows we focus on destroying SSC by magnetic fields applied parallel to the surface. In high enough fields one should expect the appearance of a $2 \mathrm{D}$ version of inhomogeneous superconducting state known as LarkinOvchinnikov-Fulde-Ferrell phase (LOFF) [8]. It was also shown that two-dimensionality broadens the region of the LOFF state on the B-T phase diagram [9]. Motivated by experimental findings mentioned above, we investigate the peculiarities introduced into this phenomenon by the SO effect or non-s-wave pairing. Experiments by Schön et al. not only have proved FET to be an effective doping process. Different levels of doping result in different $T_{c}$-s. Our results for SSC in parallel magnetic fields are expressed in terms of this $T_{c}$, providing the tool for comparing theoretical predictions with experiments by controlling the doping level. Theoretically, there is no long range order in a 2D superconductor. However, correlations are destroyed on the exponentially large spatial scale, $R \sim \xi_{0} \exp \left(E_{F} / T\right)$, which would exceed the size of the film. 
We employ below the weak-coupling BCS-like scheme by assuming that electrons interact via a weak shortranged interaction, $U\left(\boldsymbol{r}, \boldsymbol{r}^{\prime}\right)$. Then $T_{c} \ll \epsilon_{F}$, and only a narrow vicinity of the Fermi surface is involved. Thus, the interaction in the momentum representation can be taken in the form:

$$
U\left(\boldsymbol{p}, \boldsymbol{p}^{\prime}\right)=\sum_{l} U_{l} \chi_{l}(\boldsymbol{p}) \chi_{l}\left(\boldsymbol{p}^{\prime}\right),
$$

where $\boldsymbol{p}, \boldsymbol{p}^{\prime}$ lie on the Fermi surface; the angular dependence is expressed through a complete set of basis functions $\chi_{l}(\boldsymbol{p})$ (index $l$ enumerates different representations, as in expansions over the spherical functions in a 3D isotropic model). Superconducting order parameter, the "gap", $\hat{\Delta}_{\alpha \beta}(\boldsymbol{p})$ is defined by the equation:

$\hat{\Delta}_{\alpha \beta}(\boldsymbol{p})=\left|U_{l}\right| \chi_{l}(\boldsymbol{p}) \int \frac{d^{3} p^{\prime}}{(2 \pi)^{3}} \chi_{l}\left(\boldsymbol{p}^{\prime}\right)\left(T \sum_{\omega_{n}} F_{\alpha \beta}\left(\boldsymbol{p}^{\prime} ; i \omega_{n}\right)\right)$,

where $F_{\alpha \beta}\left(\boldsymbol{p}^{\prime} ; i \omega_{n}\right)$ stands for the Fourier component of Gor'kov anomalous function:

$$
\hat{F}_{\alpha \beta}\left(\boldsymbol{r}-\boldsymbol{r}^{\prime}, \tau-\tau^{\prime}\right)=-\left\langle T_{\tau}\left(\hat{\Psi}_{\alpha}(\boldsymbol{r}, \tau) \hat{\Psi}_{\beta}\left(\boldsymbol{r}^{\prime}, \tau^{\prime}\right)\right)\right\rangle,
$$

and $U_{l}<0$ is a constant in Eq.(11) corresponding to the selected pairing channel. When the field operators for electrons are re-written in momentum space, $\hat{\Psi}_{\alpha}(\boldsymbol{r}, \tau)=$ $\sum_{\boldsymbol{p}} \hat{\Psi}_{\alpha}(\boldsymbol{p}, \tau) e^{i \boldsymbol{p} \cdot \boldsymbol{r}}, \mathrm{Eq} \cdot(2)$ becomes:

$\hat{\Delta}_{\alpha \beta}(\boldsymbol{p})=\left|U_{l}\right| \chi_{l}(\boldsymbol{p}) \int \frac{d^{3} p^{\prime}}{(2 \pi)^{3}} \chi_{l}\left(\boldsymbol{p}^{\prime}\right)\left\langle\hat{\Psi}_{\alpha}\left(\boldsymbol{p}^{\prime}, \tau\right) \hat{\Psi}_{\beta}\left(-\boldsymbol{p}^{\prime}, \tau\right)\right\rangle$.

The two operators inside brackets in Eq.(4) anticommute. In the presence of the center of inversion (CI) the behavior of $\chi_{l}\left(\boldsymbol{p}^{\prime}\right)$ at $\boldsymbol{p}^{\prime} \longrightarrow-\boldsymbol{p}^{\prime}$ alone determines the symmetry of $\hat{\Delta}_{\alpha \beta}(\boldsymbol{p})$ (even (singlet) vs odd (triplet) parity pairing). For an s- or d-pairing the order parameter below $T_{c}$ has the form:

$$
\hat{\Delta}_{\alpha \beta}(\boldsymbol{p} ; \boldsymbol{q})=\Delta(\boldsymbol{q}, T)\left(i \sigma_{y}\right)_{\alpha \beta} \chi_{l}(\boldsymbol{p}),
$$

where the momentum $\boldsymbol{q}$ stands for the spatial dependence of the gap amplitude, $\Delta(\boldsymbol{q}, T)$.

Surface always breaks the CI symmetry due to the difference between the "top" and the "bottom". The direction bulk-to-surface determines $\boldsymbol{n}$, a unit vector normal to the surface. Qualitative changes in the surface electronic spectrum come about from the well-known Rashba term 10]:

$$
\hat{h}_{S O}=\alpha(\boldsymbol{\sigma} \times \boldsymbol{p} \cdot \boldsymbol{n}),
$$

which specifies SO interactions at a surface. Eq.(6) lifts the two-fold spin degeneracy for band electrons. The electron spectrum now consists of two branches with two Fermi surfaces:

$$
\epsilon_{ \pm}(\boldsymbol{p})=v_{F}\left(p-p_{F \pm}\right) ; \quad p_{F \pm}=p_{F}\left(1 \pm \frac{\alpha}{v_{F}}\right)
$$

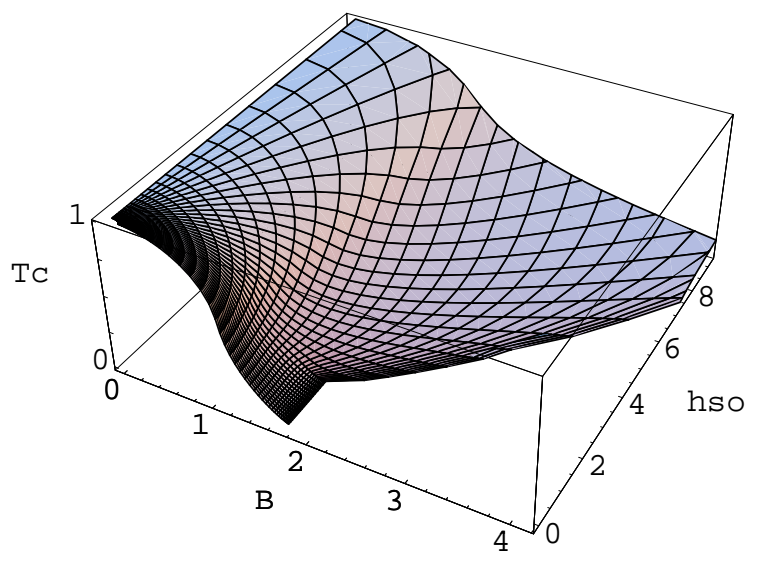

FIG. 1: Calculated $T_{c}$ as a function of magnetic field and spin-orbit interaction $\alpha p_{F}$ for a $2 \mathrm{D}$ surface superconductor. All values are in units of $T_{c 0}, T_{c}$ for the $2 \mathrm{D}$ superconductor in the absence of magnetic field. Thus, $B \rightarrow \mu_{B} B / T_{c 0}, h_{S O} \rightarrow$ $\alpha p_{F} / T_{c 0}$, and $T_{c} \rightarrow T_{c} / T_{c 0}$.

Even though SO splitting, $2 \alpha p_{F}$, may be on a scale of tenths of eV [1, 2], we assume that $2 \alpha p_{F} \ll \epsilon_{F}$. Below we speak of a strong or weak SO meaning the relative values of $2 \alpha p_{F}$ and $\mathrm{T}_{c}$.

Some theory issues regarding superconductivity without CI due to the presence of the SO term Eq.(6) were first considered in Ref. 111] and more recently in Ref. [12. The Gor'kov function (in the momentum representation), $F_{\alpha \beta}(\boldsymbol{p},+0)=-\left\langle\hat{\Psi}_{\alpha}(\boldsymbol{p}) \hat{\Psi}_{\beta}(-\boldsymbol{p})\right\rangle$, that stands under integral in Eq.(㺼) represents the wave function for Cooper pairs in the condensate. In the presence of CI symmetry the latter can be classified according to the parity:

$$
F_{\alpha \beta}(\boldsymbol{p} ;+0)=\left\{\begin{array}{c}
i\left(\sigma_{y}\right)_{\alpha \beta} f(\boldsymbol{p}) ; \quad(f(\boldsymbol{p}) \text { even }) \\
i\left[(\boldsymbol{d}(\boldsymbol{p}) \cdot \hat{\boldsymbol{\sigma}}) \hat{\sigma}_{y}\right]_{\alpha \beta} ; \quad(\boldsymbol{d}(\boldsymbol{p}) \text { odd })
\end{array}\right.
$$

With CI broken by non-zero SO term Eq.(6), the pairing wave function becomes a mixture of even and odd terms. It is important to realize that while this mixing changes physical properties of the SC phase, the gap order parameter, $\hat{\Delta}_{\alpha \beta}(\boldsymbol{p} ; \boldsymbol{q})$, preserves its singlet form Eq.(5). For instance, s-pairing indeed induces the non-zero triplet component Eq.(8), as shown in Refs. 11, 12. However, the latter does not automatically generate a "triplet" gap, $\hat{\Delta}_{\alpha \beta}^{t}(\boldsymbol{p} ; \boldsymbol{q})$. Indeed, re-writing the integration over $\boldsymbol{p}^{\prime}$ in Eq.(1) as $d^{3} p^{\prime} \rightarrow d S_{F}^{\prime} d \xi$, where $\xi=v_{F}\left(p-p_{F}\right)$, we notice that the triplet $F$-component is odd in particlehole transformation, $\xi \rightarrow-\xi$, and, hence, the integrals of the form Eq.(画) would only give small terms of or$\operatorname{der}\left(\alpha p_{F} / \epsilon_{F}\right) \ll 1$. In other words, while SO interaction may significantly change spin structure of the normal and anomalous Green functions, the "gap", $\hat{\Delta}_{\alpha \beta}(\boldsymbol{p}, \boldsymbol{q})$, Eq.(2) preserves its usual form Eq.(5) with $\chi_{l}(\boldsymbol{p})=$ const for isotropic pairing, and $\chi_{l}(\boldsymbol{p}) \propto\left(p_{x}^{2}-p_{y}^{2}\right)$ for the d-wave pairing.

We have calculated $T_{c}$ numerically for the $2 \mathrm{D}$ super- 
conductor as a function of magnetic field and spin-orbit interaction. The result is shown in Fig.1 for the magnetic field strictly parallel to the surface (to exclude diamagnetic currents) 9.

A $1^{s t}$ order phase transition was initially expected between superconducting and normal states, defined by comparing their free energies: $F_{s}(T)=F_{n}-\chi_{N} \frac{B^{2}}{2}$, which would determine the so-called paramagnetic critical field, $H_{\text {par }}$ [13] $\left(\chi_{N}\right.$ - the spin susceptibility in the normal phase). The transition from normal to superconducting state is actually (at lower temperatures) a second order transition into the LOFF state. The details of the phase diagram in the vicinity of the $H_{p a r}$ were studied numerically in Ref.14. The LOFF phase boundary is determined by Eq.(2) or Eq.(4), linearized in $\Delta_{\alpha \beta}(\boldsymbol{q})$ at an extremal $\boldsymbol{q}$. The corresponding expression for anomalous function linear in $\Delta_{\alpha \beta}(\boldsymbol{q})$ is obtained by solving the proper Gor'kov equations:

$F_{\alpha \beta}\left(\boldsymbol{p}, \boldsymbol{q} ; i \omega_{n}\right)=-\hat{G}_{\alpha \nu}^{(0)}\left(\boldsymbol{p} ; i \omega_{n}\right) \hat{\Delta}_{\rho \nu}(\boldsymbol{p}, \boldsymbol{q}) \hat{G}_{\beta \rho}^{(0)}\left(-\boldsymbol{p}+\boldsymbol{q} ;-i \omega_{n}\right)$,

where $\hat{G}_{\alpha \beta}^{(0)}\left(\boldsymbol{p} ; i \omega_{n}\right)$ is the normal state Green function at non-zero $\hat{h}_{S O}(\boldsymbol{p})$ of Eq.(6), together with the Zeeman term, $\mu_{B}(\boldsymbol{\sigma} \cdot \boldsymbol{B})$ :

$$
\left[i \omega_{n}-\xi-\hat{h}_{S O}(\boldsymbol{p})-\mu_{B} \hat{\boldsymbol{\sigma}} \boldsymbol{B}\right] \hat{G}_{\alpha \beta}^{(0)}\left(\boldsymbol{p} ; i \omega_{n}\right)=\hat{1} .
$$

The spin Hamiltionian on the left side, $\hat{H}=\hat{h}_{S O}(\boldsymbol{p})+$ $\mu_{B} \hat{\boldsymbol{\sigma}} \boldsymbol{B}$, may be easily diagonalized:

$$
\tilde{\epsilon}_{\lambda}(p)-\xi=-\lambda \sqrt{\alpha^{2} p_{F}^{2}+2 \alpha p_{F y} \mu_{B} B+\left(\mu_{B} B\right)^{2}} \equiv-\lambda \tilde{\epsilon}(p)
$$

( $\lambda= \pm 1$ for the two branches, $p_{F y}$ is the y-axis projection of the Fermi momentum, and $B \| x$ ) with the eigenfunctions, spinors $\eta^{\lambda}(\boldsymbol{p})$ of the form:

$$
\eta^{\lambda}(\boldsymbol{p})=\frac{1}{\sqrt{2}}\left\{\begin{array}{c}
1 \\
\frac{\mu_{B} B-i e^{i \varphi(\boldsymbol{p})} p_{F} \alpha}{\lambda \tilde{\epsilon}(p)}
\end{array}\right\} .
$$

Substitution of Eq.(9) into Eq.(2) making use of Eq.(5) results in a rather cumbersome expression which generalizes the corresponding Eq.(7) of Ref.9. We sketch, therefore, only a few results for the low- $\mathrm{T}$ part of the phase diagram in Fig.1. Below we discuss the main changes in the shape of the phase diagram, as introduced by SO coupling or anisotropy. As for the structure of the LOFF phase itself, we assume that the numerical analysis done in Ref. 114 remains applicable, i.e. the order parameter in the LOFF state has the structure of periodic stripes.

We state in more detail our results for the limiting cases of strong and weak SO interaction, which significantly simplify all calculations.

a) Strong $S O$ : $\alpha p_{F} \gg \Delta(0)$; subsequent analysis, which we do not provide here, leads after short calculations to our final results:

$$
\begin{aligned}
& \boldsymbol{q} \perp \boldsymbol{B}, \quad|\boldsymbol{q}|=\frac{2 \mu_{B} H_{c 2}}{v_{F}} ; \\
& \mu_{B} H_{c 2}=\sqrt{2 \Delta(0) \alpha p_{F}} \equiv \sqrt{\frac{2 \pi}{\gamma} T_{c 0} \alpha p_{F}}
\end{aligned}
$$

One sees that SO interaction not only enhances the value of $H_{c 2}$ in comparison with the LOFF for 2D model of Ref.[9] , but it also fixes the direction of the structure vector. The resulting stripe structure is parallel to the magnetic field direction and has the perpendicular space periodicity $L=\sqrt{\frac{\pi \gamma}{2 T_{c 0} \alpha p_{F}}} v_{F}$.

According to 12, in the limit of strong SO interaction the spin susceptibility for parallel fields in superconducting state, $\chi_{S}(T)$ is non-zero and equal to $\frac{1}{2} \chi_{N}$. This increases the critical paramagnetic field [13] only by a factor of $\sqrt{2}, \mu_{B} H_{\text {par }}=\Delta(0)$. Comparing this with Eq.(13), one sees that strong SO significantly increases the area occupied by the LOFF state by a factor of $\sqrt{\alpha p_{F} / T_{c 0}} \gg 1$. Strong SO scattering by defects also enhanses $H_{c 2}$ 115, but the LOFF state does not exist in presence of disorder. Analytical expressions can also be obtained at low temperatures. For the dependence of the transition temperature on magnetic field, $T_{c}(B)$, one obtains, at $T \ll T_{c 0} \sqrt{T_{c 0} /\left(\alpha p_{F}\right)}$ :

$$
T_{c}(B)=5.784\left(\sqrt{2 \pi T_{c 0} \alpha p_{F} / \gamma}-\mu_{B} B\right)^{3} /\left(\alpha p_{F}\right)^{2},
$$

and

$$
T_{c}(B)=\pi T_{c 0}^{2} /\left(2 \gamma \mu_{B} B\right), \quad T_{c 0} \sqrt{T_{c 0} /\left(\alpha p_{F}\right)} \ll T \ll T_{c 0}
$$

The expression for $T_{c}(B)$ also simplifies for small magnetic fields near $T_{c 0}$ :

$$
\frac{T_{c 0}-T_{c}}{T_{c 0}}=\frac{7 \zeta(3)}{8 \pi^{2}} \frac{\left(\mu_{B} B\right)^{2}}{T_{c 0}^{2}}
$$

Note that while the spin-orbit interaction splits the Fermi surface, quasiparticles with the same band spin index Eq.(11) form Cooper pairs in the superconducting state, so that spin-orbit interaction alone does not change $T_{c}$.

b) Weak SO: $\alpha p_{F} \ll \Delta(0)$; unlike in case of strong SO, the Cooper pair is formed mainly by pairing of electrons from the FS's with different spin indices. The LOFF phase in a $2 \mathrm{D}$ superconductor with no $\mathrm{SO}$ interaction was first analysed in Refs [9, 14]. According to Ref. [9, $\mu_{B} H_{c 2}=\Delta(0)=\sqrt{2} H_{\text {par }}, v_{F} q=2 \mu_{B} H_{c 2}$.

This result is zero order in spin-orbit interaction. The direction of $\boldsymbol{q}$ is not fixed with respect to $B$. Analysis to the second order in $\alpha p_{F}$ results in an anisotropy term which again fixes the vector $\boldsymbol{q}$, as in strong SO case Eq.(13), perpendicular to the direction of the magnetic field. Indeed, for the critical field as a function of the angle $\beta$ between $\boldsymbol{q}$ and $\boldsymbol{B}$ we find:

$$
\mu_{B} H_{c 2}=\Delta(0)-\frac{\alpha^{2} p_{F}^{2}}{2 \Delta(0)} \cos ^{2} \beta,
$$


i.e. the maximum for $H_{c 2}$ is reached for $\beta= \pm \frac{\pi}{2}$.

For small magnetic fields near $T_{c}$ we get:

$$
\frac{T_{c 0}-T_{c}}{T_{c 0}}=\frac{7 \zeta(3)}{4 \pi^{2}} \frac{\left(\mu_{B} B\right)^{2}}{T_{c 0}^{2}}
$$

A quadratic dependence on $B$ remains valid for fields $\mu_{B} B \sim \alpha p_{F}$. However, note the factor two difference between Eqs.(16) and (18): suppression of $T_{c}$ by magnetic field turns out to be slower in case of strong spin-orbit interaction than in case when the spin-orbit interaction is weak.

Although a more complicated LOFF periodic superstructure is possible, the energy considerations of Ref. \& have shown in $3 \mathrm{D}$ that the stripe phase is energetically more favorable. A detailed study done numerically in 2D 14 has shown a more complicated than just a sinusoidal shape of the order parameter. We assume that the results of Ref.14 remain valid in all cases considered above, so that the LOFF state preserves its striped order parameter form.

The major role of SO is in fixing of the LOFF superstructure. Anisotropy fixes the orientation of the LOFF stripes as well (see below). A $1^{\text {st }}$ order re-orientation transition may be expected corresponding to an abrupt change in the direction of the superconducting stripes (similar to a spin-flop transition), as the magnetic field is rotated in the $2 \mathrm{D}$ plane.

In the above discussion so far we have neglected any anisotropy at all. Meanwhile, the anisotropy is, of course, important. We address the issue of the effect of pinning stripe direction to the particular crystal axis only for the $\mathrm{d}$-wave order parameter, since the latter is intrinsically anisotropic. Stripes may orient themselves along the directions of the gap maximums. It can be easily shown that $H_{c 2}$ for the $d_{x^{2}-y^{2}}$ superconducting order parameter takes the form: $\mu_{B} H_{c 2}=\frac{\pi}{\gamma} e^{1 / 4} T_{c 0}$. The critical field for the LOFF phase of d-wave is somewhat higher than for s-wave (without the SO term Eq.(7)), as supeconducting stripes get pinned to the crystal axes by the form of the order parameter and the direction of the magnetic field. Reorientation transition/twinning is also expected for any other cause.

In summary, we have shown: i) that inhomogeneous state in parallel fields extends considerably the low temperature phase diagram of surface superconductivity with increased spin-orbit interaction; ii) all SC characteristics of the phase diagram in the $(B, T)$ plane can be expressed in terms of $T_{c 0}$, the critical temperature in the absence of the field, which is controlled by doping, and, hence, not only allows a data comparison in the broad range of FET-doping, but may also serve as a method to extract the value of the SO interaction; iii) LOFF state properties are strongly anisotropic in the plane with respect to field direction - this, for example, can be seen by measuring the anisotropy of the AC susceptibility signal; iv) the indispensible feature of the LOFF state must be the re-orientation transitions at the field rotation in the plane caused by locking of the LOFF order parameter by anisotropy.

We would like to thank L. N. Bulaevskii for useful discussions and V. M. Edelstein for a few references, including Ref.|11. This work was supported by NHMFL through the NSF Cooperative agreement No. DMR9521035 and the State of Florida.
[1] S. LaShell, B. A. McDougall, and E. Jensen, Phys. Rev. Lett. 77, 3419 (1996).

[2] E. Rotenberg, J. W. Chung, and S. D. Kevan, Phys. Rev. Lett. 82, 4066 (1999).

[3] L. Petersen et al., Phys. Rev. B 57, R6858 (1998).

[4] S. Reich and Y. Tsabba, Eur. Phys. J. B 9, 1 (1999); Y. Levi et al., Europhys. Lett. 51, 564 (2000).

[5] J.B. Goodenough, Progr. Solid State Chem. 5, 149 (1971).

[6] J. H. Schön, Ch. Kloc, R. C. Haddon, and B. Battlog, Science 288, 656 (2000); J. H. Schön, Ch. Kloc, and B. Battlog, Nature 406, 702 (2000).

[7] J. H. Schön, M. Dorget, F.C. Beuran, X.Z. Zu, E. Arushavov, C. Deville Cavellin, and M. Lagnës, Nature 414, 434 (2001).

[8] A. I. Larkin and Yu. N. Ovchinnikov, Sov. Phys. JETP 20, 762 (1962); P. Fulde, R. A. Ferrell, Phys. Rev. 135, A550 (1964).

[9] L. N. Bulaevskii, Sov. Phys. JETP 37, 1133 (1973) [ZhETF 64, 2241 (1973)]; Sov. Phys. JETP 38, 634 (1974) [ZhETF 65, 1278 (1973)]; M. Houzet, A. Buzdin, L. Bulaevskii, and M. Maley, Phys. Rev. Lett. 88, 227001
(2002).

[10] E. I. Rashba, Sov. Phys. - Solid State, 2, 1109 (1960); Yu. A. Bychkov and E. I. Rashba, Sov. Phys. - JETP Lett. 39, 78 (1984).

[11] V. M. Edelstein, Sov. Phys. JETP 68, 1244 (1989).

[12] L.P. Gor'kov and E. I. Rashba, Phys. Rev. Lett. 87, 037004 (2001) (The problem has also been treated by L.N. Bulaevskii, A.A. Guseinov, and A.I. Rusinov, Sov. Phys. JETP 44, 1243 (1976) [ZhETF 71, 2356 (1976), although their results contain errors).

[13] A. M. Clogston, Phys. Rev. Lett. 9, 266 (1962); B. S. Chandrasekhar, Appl. Phys. Lett. 1, 7 (1962).

[14] H. Burkhardt and D. Rainer, Ann. Physik 3, 181 (1994). This work claims that, instead of the 1-st order transition predicted in Ref. 13], the LOFF state transforms continuously into a uniform superconductor by developing wide regions of a constant order parameter separated by narrow domain walls where the order parameter changes sign.

[15] R. A. Klemm, A. Luther, and M. R. Beasley, Phys. Rev. B 12, 877 (1975). 